\title{
Prevalence of Depression among Transgender Male to Female in Kuantan, Pahang
}

Md Hadis ND ${ }^{1}$, Draman $\mathrm{S}^{1}$, Samsudin $\mathrm{S}^{1}$, Yusuf $\mathrm{MZ}^{2}$, Md Rosli AN ${ }^{3}$, Abd Rahman NS ${ }^{4}$

${ }^{1}$ Department of Family Medicine, International Islamic University Malaysia, Pahang, Malaysia

${ }^{2}$ Department of Community Medicine, International Islamic University Malaysia, Pahang

${ }^{3}$ Department of Psychiatry, International Islamic University Malaysia, Pahang ${ }^{4}$ Department of Pharmacy, International Islamic University Malaysia, Pahang

Introduction: Transgender is an umbrella term for persons whose gender identity, gender expression or behaviour does not conform to that typically associated with the sex to which they were assigned at birth. Transgender individuals are particularly vulnerable to mental health concerns and psychological distress. Objective: This study aims to assess the prevalence of depression and associated risk factors among transgender male to female in Kuantan, Pahang. Material and method: This was a cross-sectional study conducted among 96 transgender male to female in Kuantan, Pahang. A convenient type of sampling was used as a mean of data collection. A selfadministered validated Malay version of Beck Depression Inventory(BDI) was used to screen for depression. Result: Majority of the respondent were Malay $(82.3 \%)$, Muslim (99\%), unmarried (93.8\%) and monthly income \&ltt;RM2300 (91.7\%). Prevalence of depression among transgender male to female in Kuantan is $37.5 \%$. There is no significant association between sociodemographic data of participants and depression. Conclusion: This study shows that almost one third of transgender male to female are at risk of depression. Therefore, screening for mental health assessment is recommended so that important measures can be taken to prevent depression among this high-risk group. 